DOI 10.31489/2021No4/20-28

UDC 538.971

\title{
STUDY OF THE EFFECT OF THE CHANGE IN THE APPLIED POTENTIAL DIFFERENCE ON THE PROPERTIES OF CdSe: Ni THIN FILMS
}

\author{
Shlimas D. ${ }^{1,2}$, Omarova A. ${ }^{1}$, Kozlovskiy A.L. ${ }^{1,2 \star}$, Zdorovets M.V., ${ }^{1,3}$ \\ 1'L.N. Gumilyov Eurasian National University, Nur-Sultan, Kazakhstan, kozlovskiy.a@inp.kz \\ 2Institute of Nuclear Physics, Almaty, Kazakhstan \\ 3Ural Federal University, Yekaterinburg, Russia
}

\begin{abstract}
This work is devoted to the study of the efficiency of using the effect of nickel doping of thin films based on CdSe with the possibility of varying the structural and optical properties due to a change in the phase composition of the films. The method of electrochemical reduction of metal ions from aqueous solutions of electrolytes was chosen as a method for obtaining films. The variation of the applied potential difference was used to produce films with different characteristics. To characterize the properties and dynamics of their changes, methods of atomic force microscopy, energy dispersion analysis and X-ray diffraction were chosen. The strength mechanical properties of the films were studied using the indentation method. During experiments conducted dependencies of changes of structural, optical, strength characteristics of synthesized films depending on production conditions are obtained. Photocatalytic tests to determine the rate and efficiency of decomposition of the Rodamine $B$ organic dye showed that films in the composition of which the NiSe phase prevails have the greatest potential for use as photocatalysts.
\end{abstract}

Keywords: doping, CdSe, photocatalysis, thin films, reaction rate

\section{Introduction}

In the modern world, one of the key problems is pollution by products of the chemical and textile industries, which include various organic dyes used to color various materials [1-3]. At the same time, the majority of manufacturing products and waste during disposal retain their resistance to most physical, chemical and mechanical processing methods. One of the most common dyes used in industrial production is Rhodamine B, which allows giving the material a bright saturated color. However, despite the prevalence of this type of dyes, the processes of its utilization and processing are rather complicated, and the efficiency of processing and bringing the decomposition processes to harmless components is low with standard methods of disposal $[4,5]$. In turn, the accumulation of dye residues in aqueous media can lead to serious negative consequences associated with mutational processes or environmental pollution $[6,7]$.

To solve this problem, in recent years, methods of photocatalytic reactions with the use of various catalysts based on various semiconducting materials or ferroelectrics have been increasingly used [8-10]. The interest in these classes of materials is due to their optical properties and the band gap, which plays an important role in the processes of photodegradation and splitting of dyes under the influence of UV light. One of the promising materials is thin films based on cadmium-selenium, which have excellent semiconductor and optical characteristics, allowing them to be used as a basis for photoresistors, semiconductors, etc. [11-15]. At the same time, one of the promising areas of research in this field is the modification of thin films by doping them with magnetic elements from the iron subgroup, which make it possible to make significant changes in both the structural and optical properties of the films [16-20]. The main effect on which all changes in the properties of films are based is the replacement of cadmium or selenium with metal ions, followed by the possibility of forming substitutional or interstitial solid solutions. In this case, the formation of such phases leads not only to a change in structural changes, but also has a significant effect on an increase in the structural ordering degree in films and, consequently, a change in the strength properties, as well as an increase in resistance to external influences. It is also worth noting that, in some cases, doping with metals from the iron subgroup allows one to obtain films with grains of the «coreshell» type, which have their own specific features $[21,22]$. 
One of the promising methods for producing thin films is the method of electrochemical reduction of metal ions from aqueous solutions of electrolytes, which makes it possible to obtain films with a controlled thickness, as well as a controlled phase and elemental composition [23-25]. At the same time, this method makes it possible to quite effectively carry out various modifications of films by adding various metal salts to the electrolyte solution, which are reduced on the substrate in the case of creating an applied potential difference. In turn, varying the difference between the applied potentials leads to the possibility of controlling both the thickness of the films and the concentration of recovered metal ions by changing their recovery potentials [26-30]. In this regard, the most urgent research is to assess the possibility of using the method of electrochemical synthesis of thin CdSe films from aqueous solutions of electrolytes with the addition of nickel salts to them to obtain solid solutions of substitution or interstitiality. Based on the above, the purpose of this work is to study the effect of the applied potential difference during the electrochemical reduction of metal ions from aqueous solutions on the change in the phase composition and structural parameters of CdSe films doped with nickel. The resulting films were also used as a basis for photocatalytic decomposition of the organic dye $\mathrm{V}$.

\section{Experimental part}

The electrolyte solution used for the synthesis of thin films was obtained by dissolving the following salts in an aqueous solution in a given molar ratio: $0.5 \mathrm{M} \mathrm{CdSO}_{4}, 0.5 \mathrm{M} \mathrm{SeO}_{2}, 0.5 \mathrm{M} \mathrm{NiSO}_{4} \cdot 7 \mathrm{H}_{2} \mathrm{O}$. Varying the deposition potentials in the range from $1.0 \mathrm{~V}$ to $1.5 \mathrm{~V}$ was used to obtain films of various phase compositions. Determination of the phase composition and crystal lattice parameters was assessed by analyzing X-ray diffraction patterns obtained with a D8 Advance ECO X-ray diffractometer, Bruker. The Xray diffraction patterns were recorded in the Bragg-Brentano geometry in the angular range $2 \theta=35-75^{\circ}$, with a step of 0.03 , the spectrum acquisition time was $1 \mathrm{sec}$. To calculate the crystallographic parameters, the DiffracEVA v.4.2 and TOPAS v.4 software codes were used. The phase composition was refined using the method of full-profile analysis of the positions of the diffraction maxima and their comparison with the reference values from the PDF2-2016 database.

The band gap and optical characteristics were determined using the UV-Vis transmission spectrum analysis technique obtained on the Jena Specord-250 BU optical spectrometer.

The determination of the electrophysical characteristics and the dynamics of changes in the $\mathrm{I}-\mathrm{V}$ curves depending on the conditions for the synthesis of thin films was carried out in the range of $-1.0 \mathrm{~V}$ to $1.0 \mathrm{~V}$ using the four-contact shooting method. The measurements were monitored using an Agilent 34410A multimeter with a Hewlett Packard 66312 A power supply.

The study of the efficiency of doping thin films with nickel on the decomposition rate of the organic catalyst Rhodamine B was evaluated by carrying out a model experiment of the photocatalytic reaction under the action of ultraviolet light. Rhodamine $\mathrm{B}(30 \mathrm{ml})$ diluted in distilled water was used as a model solution. A $500 \mathrm{~W}$ xenon lamp with a $420 \mathrm{~nm}$ light filter was used as an ultraviolet source. The rate of the photocatalytic decomposition reaction was estimated by measuring the absorption curves and determining the optical density by UV-Vis spectroscopy in a given wavelength range of 400-700 $\mathrm{nm}$.

\section{Results and discussion}

Table 1 shows the results of changes in elemental analysis for the studied films obtained under various synthesis conditions. The determination of elemental analysis was carried out by analyzing the obtained energy-dispersive spectra from different areas of the samples under study and then calculating the average values. Determination of the elements distribution isotropy in the structure was evaluated by obtaining maps of the elements distribution using the mapping method in energy-dispersive analysis. According to the obtained data of the maps of the distribution of elements, it was found that the distribution of elements in the structure is isotropic, without any visible areas with a higher or lower content of elements, which indicates the uniformity of the deposition of elements and the formation of stable structural elements. According to the elemental analysis data, an increase in the potential difference from 1.0 to $1.5 \mathrm{~V}$ leads to an increase in the concentration of deposited nickel in the structure of the films from 11.5 at. $\%$ up to 26.2 and 34.6 at. \% for samples obtained at potential differences of $1.0 \mathrm{~V}, 1.25 \mathrm{~V}$, and $1.5 \mathrm{~V}$, respectively.

The determination of the phase composition and the change in its dynamics depending on the preparation conditions was carried out on the basis of the analysis of X-ray diffraction patterns obtained in the angular range $2 \theta=35-75^{\circ}$. The general view of the obtained diffraction patterns indicates the 
polycrystalline structure of the obtained films with a low degree of structural ordering and a high content of amorphous-like inclusions in the structure of the films.

Table 1. Elemental composition data for the synthesized films

\begin{tabular}{|c|c|c|c|}
\hline Applied potential difference, $\mathrm{V}$ & Cd, at. w. $\%$ & Se, at. w. \% & Ni, at.w. $\%$ \\
\hline 1.0 & $43.2 \pm 1.3$ & $45.3 \pm 2.1$ & $11.5 \pm 0.9$ \\
\hline 1.25 & $37.5 \pm 1.6$ & $36.3 \pm 1.4$ & $26.2 \pm 1.3$ \\
\hline 1.5 & $34.2 \pm 1.1$ & $31.2 \pm 1.6$ & $34.6 \pm 1.7$ \\
\hline
\end{tabular}

This nature of X-ray diffraction patterns is characteristic of cadmium-selenium-based structures obtained by electrochemical deposition in the range of potential differences of $1.0-1.5 \mathrm{~V}$, characteristic of the production of amorphous films [Fig.1]. According to the obtained X-ray diffraction data, peaks characteristic of two different phases are present on diffraction patterns. According to the data of the Rietveld full-profile analysis method, these reflections are characteristic for the following phases: the hexagonal CdSe phase with the spatial system P63mc (186) and the orthorhombic NiSe phase with the spatial system R3m(160). The distorted shape of these diffraction lines, as well as their large half-widths, indicate that the structure of the films contains rather fine grains, with a strongly distorted and deformed shape.

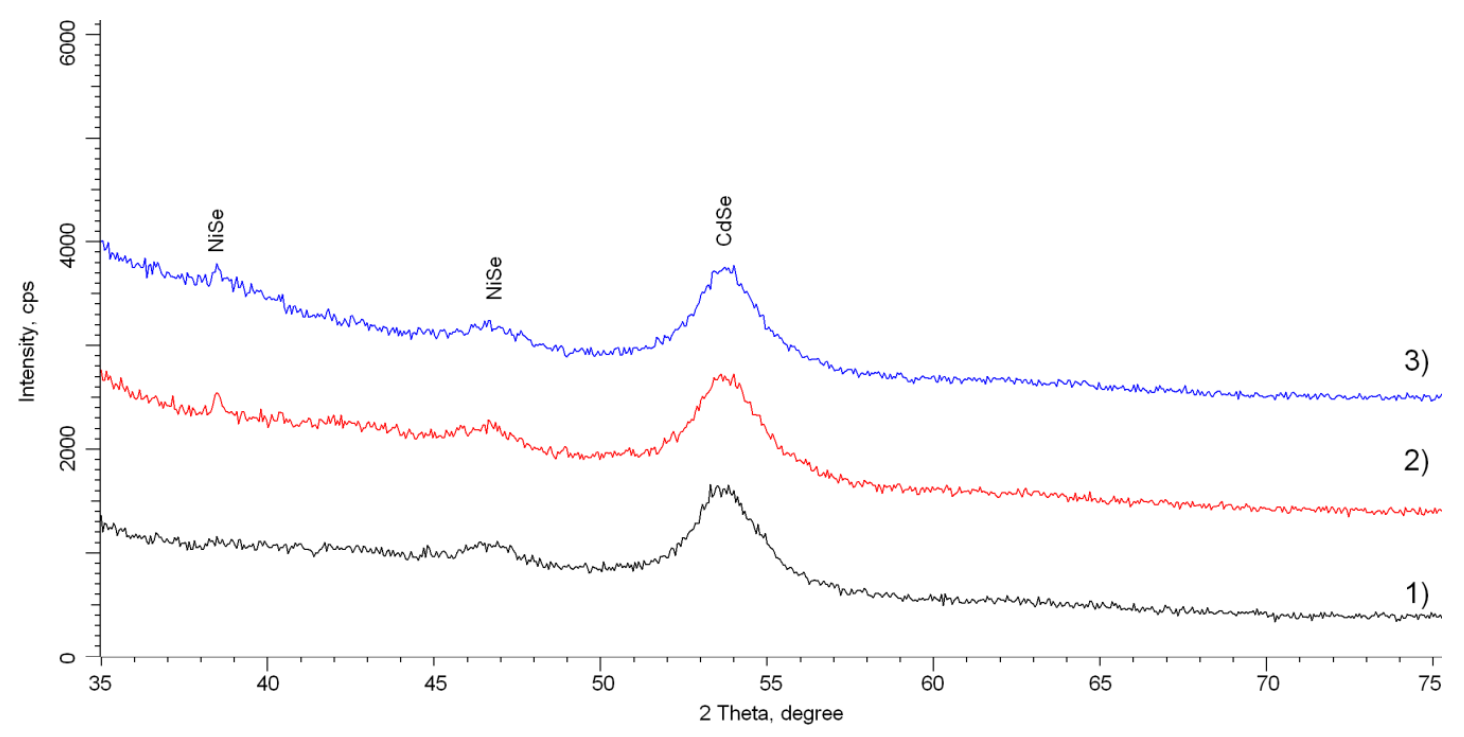

Fig.1. X-ray diffraction patterns of the synthesized films obtained at different potential differences: 1) $1.0 \mathrm{~V}$; 2) $1.25 \mathrm{~V}$; 3) $1.5 \mathrm{~V}$

Estimating the contributions of two phases depending on the applied potential difference, the dependence of the change in the phase composition of the films was plotted, which is shown in Figure 2. In the case of samples obtained at a potential difference of $1.0 \mathrm{~V}$, the NiSe:CdSe phase ratio is approximately 3:1. An increase in the applied potential difference, which leads to an increase in the concentration of nickel in the structure of the films, leads to an increase in the contribution of the NiSe phase, and to a change in the phase ratio. The increase in the contribution of the NiSe phase is due to the fact that with an increase in the applied potential difference, the rate of recovery of nickel ions from aqueous solutions of electrolytes becomes higher, as it approaches the value of the nickel reduction potential, as a result of which cadmium is partially replaced in the structure of the CdSe phase with the subsequent formation of a new NiSe phase. In this case, an increase in the potential difference leads not only to an increase in the contribution of the NiSe phase, but also to an increase in the structural ordering degree, which is expressed in a change in the shape and width of diffraction peaks characteristic of both phases.

Table 2 shows the results of changes in the crystal lattice parameters depending on the synthesis conditions at various potential differences. As can be seen from the data presented, a change in the applied potential difference leads to a decrease in the crystal lattice parameters and its volume, which indicates an increase in the structure ordering during the synthesis, as well as a decrease in the deformation contributions. 


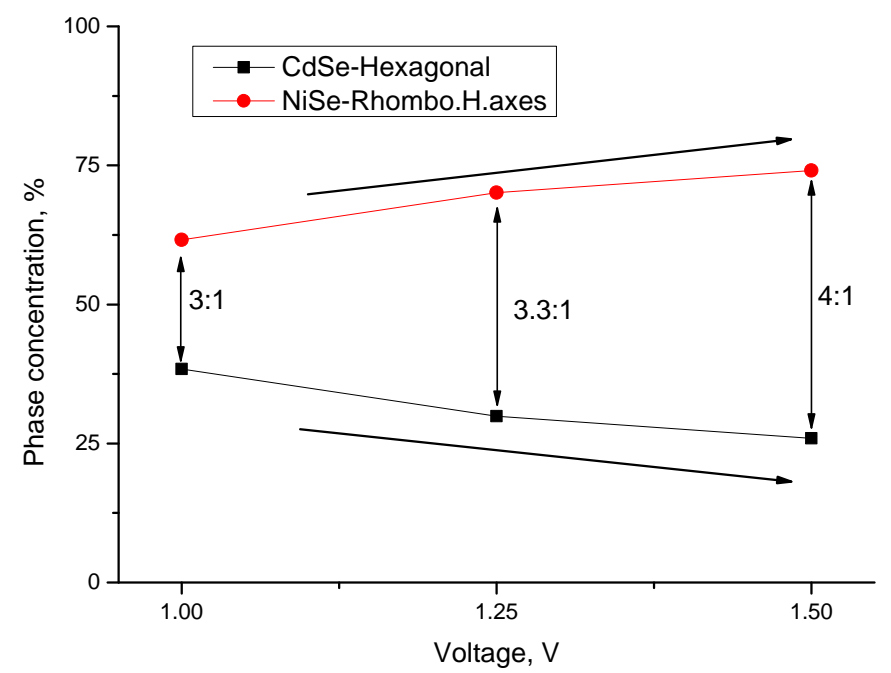

Fig.2. Diagram of changes in the films phase composition depending on the applied potential difference

It should be noted that a change in the phase composition, leading to the displacement of the hexagonal CdSe phase, also leads to an increase in the crystallinity degree by $7.6 \%$ and $12.3 \%$ for the samples obtained at a potential difference of $1.25 \mathrm{~V}$ and $1.5 \mathrm{~V}$, respectively. This behavior of changes in the structural ordering degree indicates an improvement in the structure, as well as the formation of a denser lattice and a decrease in porous inclusions, which is also evidenced by a decrease in the volume of the crystal lattice and, consequently, an increase in density.

Table 2. Crystalline parameter data

\begin{tabular}{|c|c|c|c|}
\hline $\begin{array}{l}\text { Applied potential } \\
\text { difference, } \mathrm{V}\end{array}$ & Phase & $\begin{array}{c}\text { Crystal lattice parameter, } \\
\AA\end{array}$ & $\begin{array}{c}\text { Crystallinity } \\
\text { degree, } \%\end{array}$ \\
\hline \multirow{2}{*}{1.0} & $\begin{array}{l}\text { CdSe-Hexagonal, } \\
\text { P63mc(186) }\end{array}$ & $\begin{array}{c}\mathrm{a}=4.23132, \mathrm{c}=6.91710, \\
\mathrm{~V}=107.25 \AA^{3}\end{array}$ & \multirow[t]{2}{*}{67.4} \\
\hline & $\begin{array}{c}\text { NiSe - Rhombo.H.axes } \\
\text { R3m(160) }\end{array}$ & $\begin{array}{c}a=9.99234, c=3.30785, \\
V=286.03 \AA^{3}\end{array}$ & \\
\hline \multirow{2}{*}{1.25} & $\begin{array}{l}\text { CdSe-Hexagonal, } \\
\text { P63mc(186) }\end{array}$ & $\begin{array}{c}\mathrm{a}=4.21224, \mathrm{c}=6.91452 \\
\mathrm{~V}=106.29 \AA^{3}\end{array}$ & \multirow[t]{2}{*}{75.0} \\
\hline & $\begin{array}{c}\text { NiSe - Rhombo.H.axes } \\
\text { R3m(160) }\end{array}$ & $\begin{array}{c}\mathrm{a}=10.03348, \mathrm{c}=3.31598 \\
\mathrm{~V}=289.01 \AA^{3}\end{array}$ & \\
\hline \multirow{2}{*}{1.5} & $\begin{array}{l}\text { CdSe-Hexagonal, } \\
\text { P63mc(186) }\end{array}$ & $\begin{array}{c}a=4.19159, c=6.90489, \\
V=105.06 \AA^{3}\end{array}$ & \multirow[t]{2}{*}{79.7} \\
\hline & $\begin{array}{c}\text { NiSe - Rhombo.H.axes } \\
\text { R3m(160) }\end{array}$ & $\begin{array}{c}\mathrm{a}=10.05905, \mathrm{c}=3.31953, \\
\mathrm{~V}=290.88 \AA^{3}\end{array}$ & \\
\hline
\end{tabular}

Figure 3 shows the results of changes in the morphological features of the surface relief of the films synthesized under different conditions. The general view of the changes indicates that an increase in the potential difference leads to a change in both the grain size and their concentration and agglomeration on the surface, which is primarily associated with a change in the grain formation rate, as well as a change in the phase composition and structural ordering degree.

Figure 4 shows the results of changes in the roughness and microhardness of the synthesized films depending on the applied potential difference during deposition. The roughness results were obtained by analyzing the images of the film surface obtained using atomic force microscopy. 


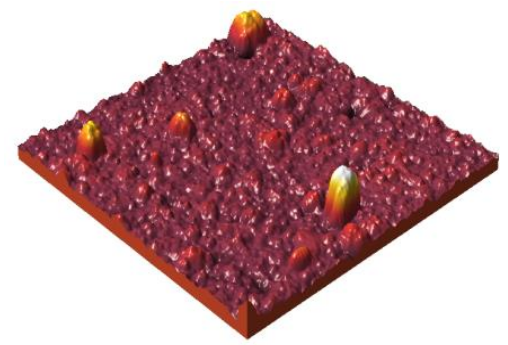

a)

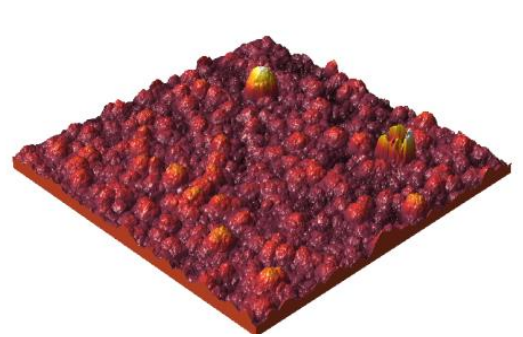

b)

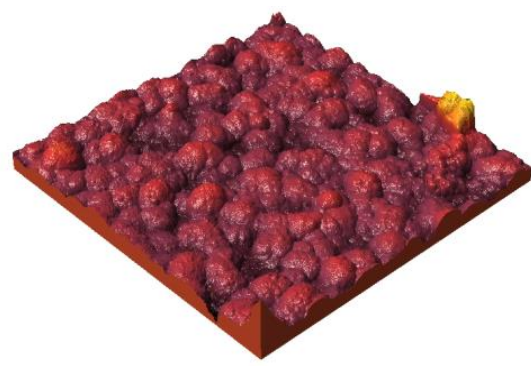

c)

Fig.3. 3D images of morphological features of synthesized films: a) $1.0 \mathrm{~V}$; b) $1.25 \mathrm{~V}$; c) $1.5 \mathrm{~V}$

An increase in the applied potential difference from 1.0 to $1.25 \mathrm{~V}$, as can be seen from the presented data, leads to an insignificant increase in the roughness degree from $11 \mathrm{~nm}$ to $26 \mathrm{~nm}$. This increase is due to a change in the size of the grains of which the film consists due to their enlargement. At the same time, an increase in the applied potential difference to $1.5 \mathrm{~V}$ leads to an increase in the roughness degree by more than a factor of 5 compared with the analogous value for the samples obtained with an applied potential difference of $1.0 \mathrm{~V}$. At the same time, the analysis of changes in morphological features showed that changes in the synthesis conditions lead not only to enlargement of grains and, consequently, to an increase in the degree of roughness, but also to an increase in the degree of grain size uniformity.

So, at a potential difference of $1.0 \mathrm{~V}$, the grain sizes, according to atomic force microscopy data, are no more than 5-10 nm, while the formation of single grains, the size of which is several tens of nanometers, is observed. An increase in the applied potential difference to $1.25 \mathrm{~V}$ leads to an increase in the grain size to 20-30 nm, as well as a decrease in the number of single large grains. At an applied potential difference of 1.5 $\mathrm{V}$, the grain sizes increase to $50-70 \mathrm{~nm}$, while the formation of large single grains in the films structure is practically not observed, which indicates an increase in the homogeneity of the films.

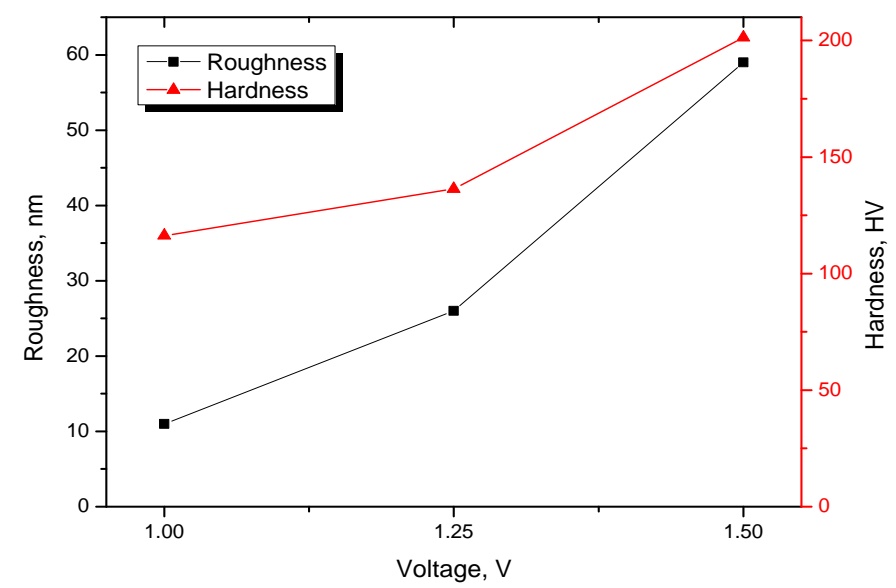

Fig.4. Graph of change of roughness and microhardness of synthesized films depending on the applied potential difference

The results presented of the change in the films microhardness depending on the preparation conditions indicate that a change in both morphological features, due to the coarsening of grains and an increase in the crystallinity degree, leads to an increase in the strength and hardness of the films. This behavior of changes in strength characteristics is due to the porosity decrease processes and the disordering regions content decrease in the films structure. In this case, a change in the potential difference to $1.25 \mathrm{~V}$ and $1.5 \mathrm{~V}$ leads to an increase in hardness by $17.2 \%$ and $73.1 \%$ in comparison to the samples obtained at a potential difference of $1.0 \mathrm{~V}$. Figure 5 shows the results of the change in the graphs of the I-V characteristics of the studied thin films, filmed in the range from -1.0 to $1.0 \mathrm{~V}$, which are characterized by the presence of two sections of the $\mathrm{I}-\mathrm{V}$ characteristic, obeying the ohmic nature $(-1.0$ to 0$) \mathrm{V}$ and the quadratic dependence of the I-V characteristic (from 0 to $1.0 \mathrm{~V}$ ). As is known, the quadratic dependence of the change in the $\mathrm{I}-\mathrm{V}$ characteristic is most characteristic of semiconductor compounds and is characterized by a limited filling of electron traps, which leads to a sharp increase in conductivity. The analysis of the dependences obtained for 
the structures under study showed that an increase in the NiSe phase contribution and the structural ordering degree leads to an increase in the conductivity and a decrease in the resistance. The increase in conductivity is also due to a decrease in the concentration of amorphous inclusions in the structure of the films, as well as to an increase in the grain size, which is accompanied by a decrease in the dislocation density and defect structure.

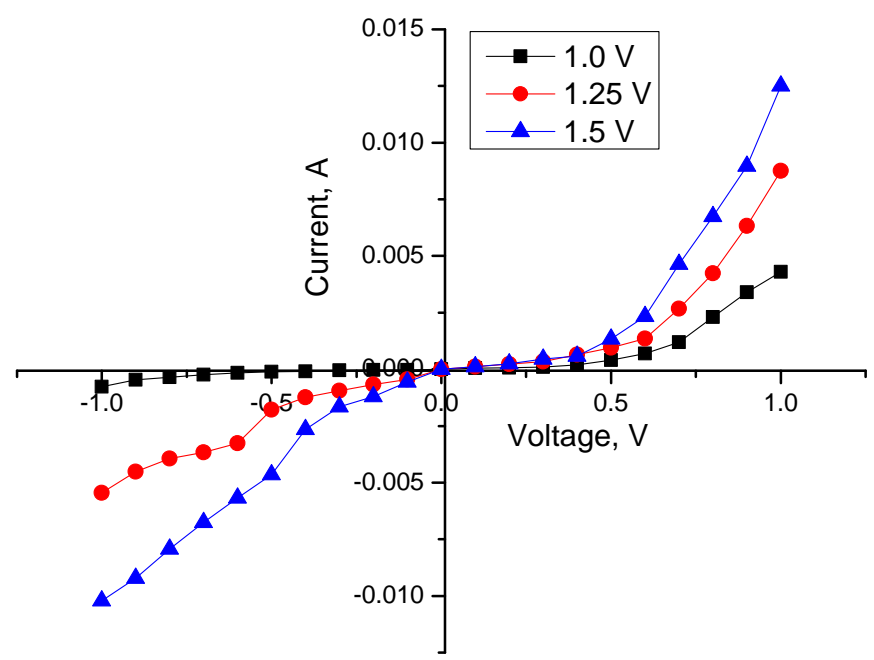

Fig.5. I -V characteristics of synthesized films depending on preparation conditions

Figure 6 shows the results of the change in the fundamental absorption edge shift value in the energy representation, which makes it possible to determine the band gap, as well as its changes depending on the phase composition of the films. The general view of changes in the fundamental absorption edge indicates that a change in the phase composition leads to a shift of the edge to the low-energy region, which indicates a decrease in the band gap. The presented dependences of the change in the band gap and the refractive index, which characterizes the optical density of the material, reflect the inverse dependence of the change in these values from each other. In this case, a change in the phase composition, as well as a decrease in the crystalline porosity, leads to an increase in the refractive index and, consequently, to a decrease in the porosity of the films. In turn, an increase in the NiSe phase contribution leads to a decrease in the band gap and, hence, to a change in the electron density of the films.

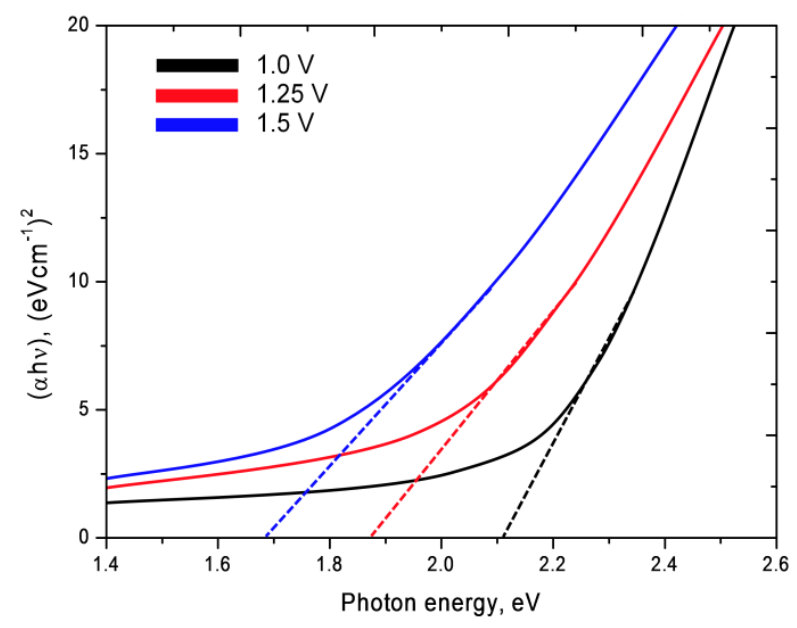

a)

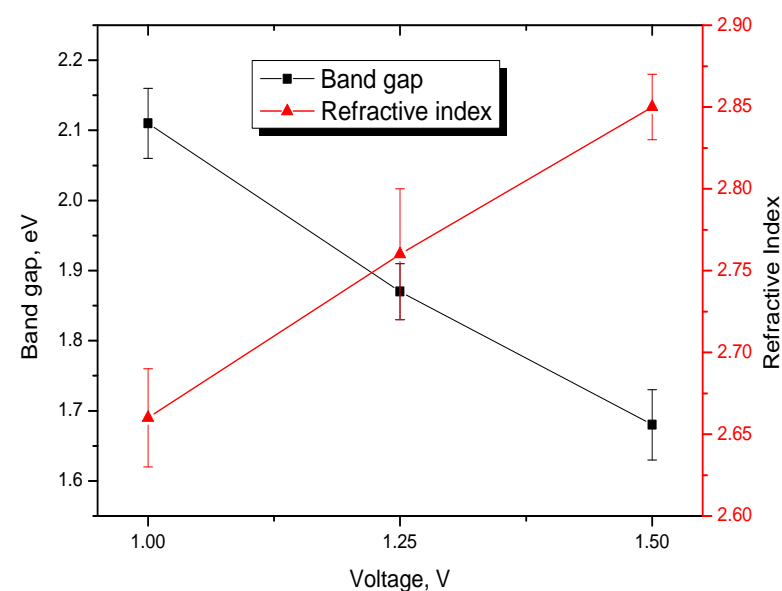

b)

Fig.6. a) Tauc' plots; b) Results of change in the band gap and refractive index

As is known, one of the promising applications of thin films is the photocatalytic decomposition of organic dyes under the action of ultraviolet radiation in the presence of catalysts. In this case, the key factors affecting the efficiency assessment of the use of certain photocatalysts are such indicators as the degradation degree and the decomposition rate of the dye in the presence of a catalyst. Also, an important role is played 
by the degree of catalyst resistance to long-term use and performance retention as a result of several cyclic tests. Figure 7 shows the results of changes in the time dependence of the Rhodamine B degradation efficiency during the photocatalytic reaction during interaction with a catalyst in the form of films obtained under different synthesis conditions. The experiment time was limited by the achievement of the maximum decomposition efficiency for one of the selected systems with a catalyst. As can be seen from the data presented in Figure 7, the highest efficiency of the decomposition of Rhodamine B during the photocatalytic reaction is possessed by films obtained at a potential difference of $1.5 \mathrm{~V}$, which amounted to more than $95 \%$ of the decrease in the optical density of Rhodamine B in the time allotted for the experiment. In the case of films obtained at potential differences of $1.0 \mathrm{~V}$ and $1.25 \mathrm{~V}$, the efficiency of photocatalytic degradation of Rhodamine B was $57 \%$ and $78 \%$, respectively.

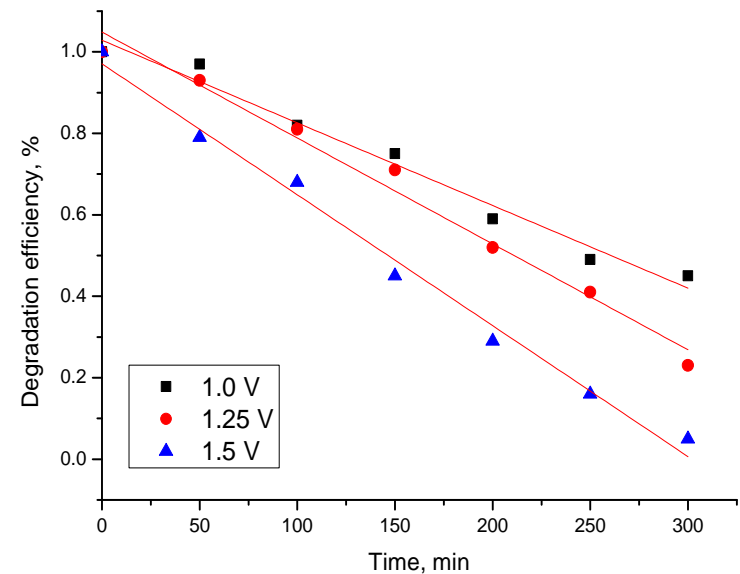

a)

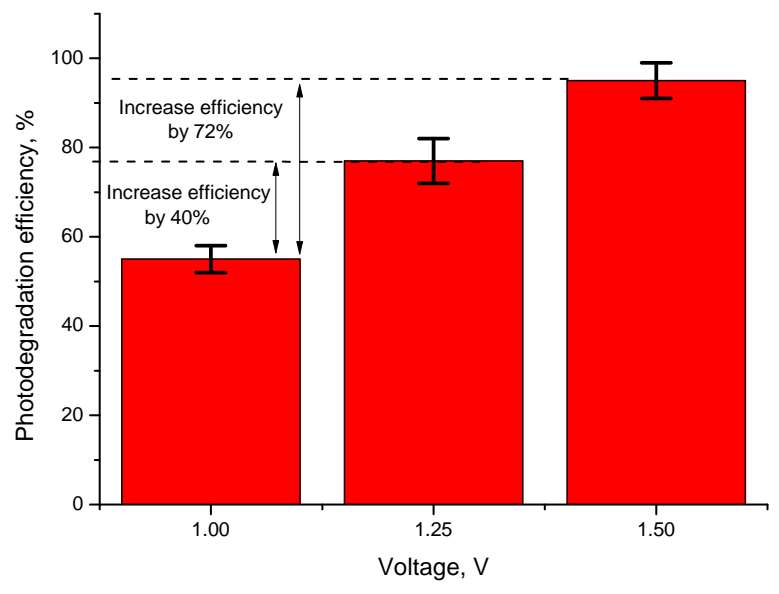

b)

Fig.7. a) Plot of time dependence of Rhodamine B degradation efficiency in case of decomposition reaction;

b) Diagram of assessment of decomposition efficiency in the final stage of photocatalytic reaction

Figure 8 shows the results of the assessment of the change in dye concentration ratio before and after the reaction on a logarithmic scale, which allows determination of the reaction rate constant. According to the calculations obtained, it was found that a change in the phase composition of the synthesized films leads to an increase in the decomposition rate by 1.5 and 3 times for samples obtained at potential differences of $1.25 \mathrm{~V}$ and $1.5 \mathrm{~V}$.

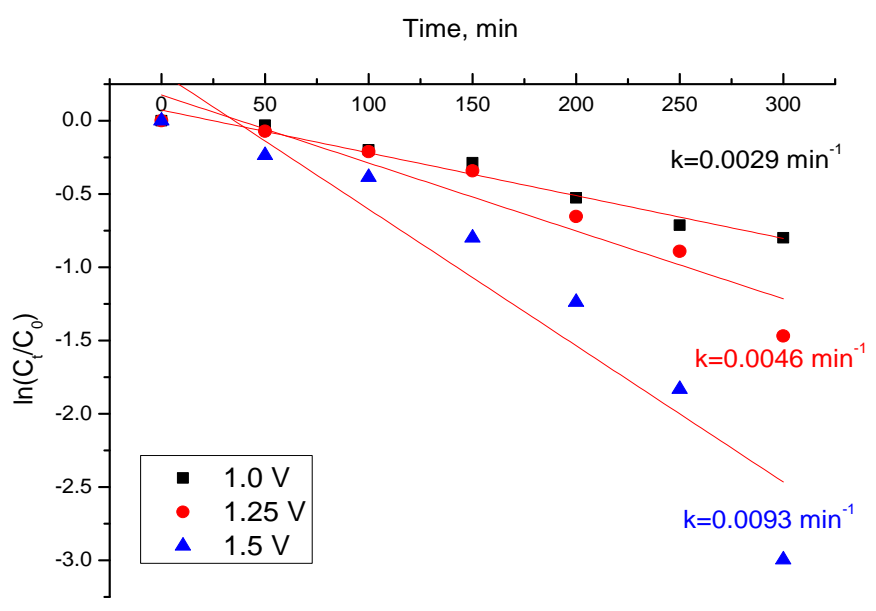

Fig.8. Data on the change in the rate constant of the Rhodamine B decomposition photocatalytic reaction

Such an increase in the degradation rate, as well as an increase in the decomposition efficiency, is caused by a change not only in the phase composition of the films, but also primarily by a change in the band gap, a decrease in which leads to an increase in the yield of electrons and photons under the action of photoionization of UV light. The photo-degradation process can be schematically written as follows: 


$$
\begin{gathered}
\mathrm{CdSe} / \mathrm{Ni} \text { films }+\mathrm{h} v \rightarrow \mathrm{e}^{-}+\mathrm{h}^{+} \\
\mathrm{H}_{2} \mathrm{O}+\mathrm{h}^{+} \rightarrow \cdot \mathrm{OH}+\mathrm{H}^{+} \\
\mathrm{OH}-+\mathrm{h}^{+} \rightarrow{ }^{\circ} \mathrm{OH} \\
\text { Dye }+{ }^{\cdot} \mathrm{OH} \rightarrow \text { oxidation products } \\
\text { Dye }+\mathrm{h}^{+} \rightarrow \text { oxidation products }
\end{gathered}
$$

Exposure to UV radiation on the sample leads to the formation of an electron and a photon, which are knocked out of the catalyst surface and, when interacting with water, form a number of ${ }^{\circ} \mathrm{OH}$ radicals that directly participate in the decomposition of the dye during the initiation of decarboxylation and hydroxylation processes with the formation of harmless components.

\section{Conclusion}

During the studies, the dependences of the effect of the applied potential difference on the change in the phase composition, structural features, and the ordering degree in nickel-doped CdSe films were established. It has been determined that an increase in the applied potential difference leads to an increase in the NiSe phase contribution formed during the substitution of cadmium ions by nickel ions during the formation of the crystal structure. It has been determined that an increase in the NiSe phase contribution leads to an increase in the structural ordering degree and hardening of the film structure. During the studies of the optical properties, it was determined that a change in the phase composition leads to a decrease in the band gap, as well as an increase in the optical density of the films. During the evaluation of the applicability of the synthesized films as a basis for photocatalysts, it was found that films with a high NiSe phase content have a high photocatalytic reaction rate as well as a high degree of photocatalytic decomposition of organic dyes.

In conclusion, the selected synthesis conditions, as well as the ratio of the starting salts, make it possible to obtain films with a controlled phase and elemental composition, which are highly promising as a basis for photocatalysts.

\section{Funding}

This research was funded by the Science Committee of the Ministry of Education and Science of the Republic of Kazakhstan (No. AP09562582).

\section{REFERENCES}

1 Liu Hao, et al. Enhanced photocatalytic capability of zinc ferrite nanotube arrays decorated with gold nanoparticles for visible light-driven photodegradation of rhodamine B. Journal of materials science. 2016, Vol. 51, No.12, pp. $5872-5879$.

2 Alshammari A., Bagabas A., and Assulami M. Photodegradation of rhodamine B over semiconductor supported gold nanoparticles: the effect of semiconductor support identity. Arabian Journal of Chemistry. 2019, Vol. 12, No. 7, pp. $1406-1412$.

3 Sangami G., Dharmaraj N. UV-visible spectroscopic estimation of photo-degradation of rhodamine-B dye using tin (IV) oxide nanoparticles. Spectrochimica Acta Part A: Molecular and Biomolecular Spectroscopy. 2012, Vol.97, pp. $847-852$.

4 Parvaz S., Rabbani M., Rahimi M. Fabrication of novel magnetic $\mathrm{ZnO}$ hollow spheres/pumice nanocomposites for photodegradation of Rhodamine B under visible light irradiation. Materials Science and Engineering: B. 2021, Vol 263 pp. 114863.

5 Kozlovskiy A.L., Alina A., Zdorovets M.V. Study of the effect of ion irradiation on increasing the photocatalytic activity of WO 3 microparticles. Journal of Materials Science: Materials in Electronics. 2021, Vol. 32, No. 3, pp. $3863-3877$.

6 Kozlovskiy A., et al. The study of the applicability of ionizing radiation to increase the photocatalytic activity of TiO 2 thin films. Journal of Nanostructure in Chemistry. 2020, Vol. 10, No. 4, pp. 331 - 346.

$7 \mathrm{He}$ M. Q., et al. Synthesis of molecularly imprinted polypyrrole/titanium dioxide nanocomposites and its selective photocatalytic degradation of rhodamine B under visible light irradiation. Express Polymer Letters. 2014, Vol 8, No. 11, pp 850-861. 
8 Larowska D. et al. Graphene oxide functionalized with cationic porphyrins as materials for the photodegradation of rhodamine B. The Journal of Physical Chemistry C. 2020, Vol. 124, No. 29, pp. 15769-15780.

9 Jadhav S.A., et al. Magneto-structural and photocatalytic behavior of mixed Ni-Zn nano-spinel ferrites: visible light-enabled active photodegradation of rhodamine B. Journal of Materials Science: Materials in Electronics. 2020 , Vol. 31, pp. 11352-11365.

10 Fattahimoghaddam H., Mahvelati-Shamsabadi T., Lee B.-K. Efficient photodegradation of rhodamine B and tetracycline over robust and green g-C3N4 nanostructures: supramolecular design. Journal of Hazardous Materials. 2020, Vol. 403, pp. 123703.

11 Il'chuk, G. A., et al. Peculiarities of the optical and energy properties of thin CdSe films. Optics and spectroscopy. 2020, Vol. 128, No. 1 pp. 49-56.

12 Milonakou-Koufoudaki, K., et al. Natural dyes in hybrid chalcogenide multi-layer thin films. Bulletin of Materials Science. 2020, Vol. 43, No. 1, pp. 1 - 11.

13 Hamann, Kathryn R., et al. Optically tunable mesoscale CdSe morphologies via inorganic phototropic growth. Journal of Materials Chemistry C. 2020, Vol. 8, No. 36, pp. 12412-12417.

14 Chauhan P., et al. Superior electrochemical activity of CdSe thin film by chromium substitutional doping. Journal of Alloys and Compounds. 2021, Vol. 862, pp. 158016.

15 Shelke N.T., Karle S.C., Karche B.R.. Photoresponse properties of CdSe thin film photodetector. Journal of Materials Science: Materials in Electronics. 2020, Vol. 31, No. 18, pp. 15061-15069.

16 Lohitha B., Thanikaikarasan S., Roji Marjorie S. Growth and characterization of CdS and Fe doped CdS thin films through electrochemical route. Materials Today: Proceedings. 2020, Vol. 33, pp. 3068-3071.

17 Patil N.M., et al. Structural, optical and electrical properties of spray-deposited Fe-doped nanocrystalline $\mathrm{ZnS}_{0.2} \mathrm{Se}_{0.8}$ thin films. Phase Transitions. 2021, Vol. 94, No. 6-8, pp. 493-510.

18 Han Chong et al. Carbon Dots Doped with $\mathrm{Ni}(\mathrm{OH})_{2}$ as Thin-Film Electrodes for Supercapacitors." ACS Applied Nano Materials. 2020, Vol. 3, No.12, pp. 12106-12114.

19 Abdulwahab A.M., Asma'a Ahmed A.L.-A., Ahmed A.A.A. Influence of Ni-Co dual doping on structural and optical properties of CdSe thin films prepared by chemical bath deposition method. Optik. 2021, Vol. 236 , pp. 166659.

20 Tan, Wenyu, et al. Nucleation and growth mechanisms of an electrodeposited $\mathrm{Ni}-\mathrm{Se}-\mathrm{Cu}$ coating on nickel foam. Journal of Colloid and Interface Science. 2021, Vol. 600, pp. 492-502.

21 Omarova, A., et al. Study of the influence of synthesis conditions on stoichiometry and the properties of nanostructured CdSe thin films. Journal of Materials Science: Materials in Electronics. 2020, Vol. 31, No. 15, pp. 12756-12764.

22 Alasvand A., Kafashan $\mathrm{H}$. Investigation the effect of $\mathrm{Pb}$ incorporation on the surface characterizations of electrodeposited CdSe nanostructures. Journal of Alloys and Compounds. 2020, Vol. 817, pp. 152711.

23 Ayal, A.K., et al. Sensitization of TiO2 nanotube arrays photoelectrode via homogeneous distribution of CdSe nanoparticles by electrodeposition techniques. Journal of Physics and Chemistry of Solids. 2020, Vol. 153, pp. 110006.

24 Shaikh A.V., et al. Electrodeposition of n-CdSe/p-Cu2Se heterojunction solar cells. Engineered Science. 2020, Vol. 13, pp. 79-86.

25 Saha S., et al. Electrodeposition Fabrication of Chalcogenide Thin Films for Photovoltaic Applications. Electrochem. 2020, Vol. 1, No.3, pp. 286-321.

26 Gullu H.H., et al. Temperature effects on optical characteristics of CdSe thin films. Materials Science in Semiconductor Processing. 2021, Vol. 123, pp. 105559.

27 Erturk K., et al. Investigation of structural, spectral, optical and nonlinear optical properties of nanocrystal CdS: Electrodeposition and Quantum Mechanical Studies. Optik. 2021, Vol. 243, pp. 167469.

28 Thanikaikarasan S., et al. Physical, chemical and optical properties of CdSe and CdSe: Zn thin films obtained through low cost electrochemical route. Materials Today: Proceedings. 2020, Vol. 21, pp. 73-77.

29 Zhang Y., et al. Alkaline electrolyte: toward high-quality CdTe films with the assistance of strong complexing agent and organic base. CrystEngComm. 2018, Vol. 20, No. 1, pp. 8-11.

30 Zyoud A., et al. Electrochemically and chemically deposited polycrystalline CdSe electrodes with high photoelectrochemical performance by recycling from waste films. Materials Science in Semiconductor Processing. 2020, Vol. 107, pp. 104852. 